\title{
EL ESTUDIO DE DROGAS EN MÉXICO: MAPEO SOBRE LA PRODUCCIÓN ACADÉMICA EN SCOPUS, WEB OF SCIENCE, PSYCINFO, SCIENCE DIRECT Y REDALYC
}

\author{
THE STUDY OF DRUGS IN MEXICO: A MAPPING OF THE ACADEMIC \\ PRODUCTION IN SCOPUS, WEB OF SCIENCE, PSYCINFO, SCIENCE DIRECT \\ AND REDALYC
}

Daniel Francisco Beltrán Velarde

Universitat Autònoma de Barcelona

\section{Abstract}

Mexico is in 2018 in a conjuncture regarding to its drug policy; several events suggest the importance of studying the correspondence between the academic production of drugs and the mexican context. Objective. To identify and analize the academic production published from 2013 to 2018 in Scopus, Web of Science, PsycINFO, Science Direct, Redalyc; and compare the results with some contextual characteristics of Mexico. Topic development. A systematic search was carried out in the databases. Then, through a document analysis, the corpus of data was classified by population, places, drugs, topics, methodologies and author affiliation. By means of a relational analysis of the categories, tendencies were identified. Conclussions. Tendencies were compared with the Encuesta Nacional de Consumo de Drogas, Alcohol y Tabaco 2016-2017, the Sistema de Vigilancia Epidemiológica de las Adicciones 2016 report and other contextual characteristics of Mexico, such as the inminent legalization of cannabis. The results suggest the need of proposing topics that attend the inminent legalization of substances such as cannabis.

Keywords: Drugs, mapping, Mexico, academic production

\section{Resumen}

México se encuentra en el 2018 ante una coyuntura respecto a su política de drogas; diversos acontecimientos sugieren la importancia de estudiar la correspondencia entre la producción académica sobre drogas y el contexto mexicano. Objetivos. Identificar y analizar los trabajos publicados del 2013 al 2018 en Scopus, Web of Science, PsycINFO, Science Direct, Redalyc; y compararlos con algunas características contextuales de México. Desarrollo del tema. Se realizó una búsqueda sistematica en las bases de datos mencionadas. Luego, mediante un análisis de documentos, se clasificó el corpus de datos por poblaciones, lugares, drogas, temas, metodologías y afiliación de autoras y autores. Posteriormente, por medio de un análisis relacional de las investigaciones, se identificaron tendencias de estudio. Conclusiones. Se compararon las tendencias de estudio con la información contenida en la Encuesta Nacional de Consumo de Drogas, Alcohol y Tabaco 2016-2017, el reporte del Sistema de Vigilancia Epidemiológica de las Adicciones 2016 y otras características del contexto mexicano, como la inminente legalización del cannabis. Los resultados sugieren la necesidad de plantear temas que atiendan la inminente regulación de determinadas drogas.

Palabras clave: Drogas, mapeo, México, producción académica.

Correspondencia: Daniel Francisco Beltrán Velarde

dani.beltran.velarde@gmail.com 
México se encuentra en la segunda mitad del 2018 ante una coyuntura en cuanto a su política de drogas se refiere: el presidente electo, que toma el cargo el 1ro de diciembre del año en cuestión, se ha manifestado por un cambio en la estrategia de seguridad del país estrechamente ligada con la política de drogas(Expansión, 2018), en busca de un proceso de reconciliación y pacificación que permita reducir los altos índices de violencia registrados desde el emprendimiento de la llamada guerra contra el narcotráfico ${ }^{1}$ en 2006 . Parte de su equipo de transición, la futura secretaria de Gobernación y el futuro secretario de Seguridad Pública, ha expresado la necesidad de despenalizar las drogas en el país (Reséndiz, 2018) y de legalizar los usos médicos y lúdicos del cannabis (Miranda, 2018). Aunado a esto, la Suprema Corte de Justicia de la Nación ha otorgado hasta el momento cuatro amparos que avalan a particulares el uso lúdico -y sus actividades correlativas- del cannabis ${ }^{2}$, instando, además, al Poder Legislativo al "diseño de una política pública integral" en esta materia (Suprema Corte de Justicia de la Nación, 2018).

Esta coyuntura puede entenderse en relación, además de con la crisis de seguridad mencionada, con otros resultados del prohibicionismo ${ }^{3}$. Este modelo no sólo ha probado ser ineficiente en cuanto a la reducción de los índices de consumo en el país ${ }^{4}$ (Comisión Nacional Contra las Adicciones-Instituto Nacional de Psiquiatría Ramón de la Fuente Muñiz, 2017), sino que también ha generado riesgos para los usuarios: violaciones de derechos humanos y la obstaculización a políticas desde la perspectiva de la salud pública -como las de reducción de riesgos y daños- (París, 2013). Así pues, el posible cambio en la manera de entender/gestionar a las drogas no debe entenderse/interpretarse únicamente a partir de la búsqueda de la disminución de los índices de violencia del país, sino también, a partir del reconocimiento de que los usuarios -más allá de la forma en que se interpreten sus consumos- tienen derecho a la salud, a la seguridad y a la libertad.
Ante este panorama, el presente artículo tiene como objetivo hacer un mapeo de las investigaciones sobre drogas y México, a manera de conocer si existe correspondencia entre éstas y las circunstancias contextuales del país. Vale precisar que, los trabajos utilizados para este mapeo tienen que ver exclusivamente con los aspectos relacionados directamente con el consumo, los procesos de rehabilitación y la política de drogas, por lo que no se incluyeron trabajos que remitieran a la violencia generada en el marco de la guerra contra el narcotráfico o a los procesos de producción, distribución y comercialización de drogas en México. Se llevó a cabo una búsqueda sistemática en cinco bases de datos: Scopus; Web of Science; PsycINFO; Science Direct; y Redalyc. Posteriormente, se condujó un análisis de los documentos que cumplieron con los criterios de inclusión y se presentaron los resultados. Por último, se discutieron dichos resultados con las características contextuales del país -presentadas a partir de la información encontrada tanto en la Encuesta Nacional de Consumo de Drogas, Alcohol y Tabaco 2016-2017 (ENCODAT 2016), como en el informe del Sistema de Vigilancia Epidemiológica de las Adicciones 2016 (SISVEA 2016) y la coyuntura descrita anteriormente-, se propusieron algunas líneas de investigación necesarias a manera de conclusiones y se reconocieron algunas limitaciones del estudio.

\section{MÉTODO}

Se hizo una búsqueda de artículos, libros, capítulos de libros y tesis publicados en cinco bases de datos, del 2013 a la fecha en que se realizaron las búsquedas. Los documentos se buscaron de manera que se encontraran aquellos que contuvieran los términos: $\operatorname{droga(s)} 0$ sustancia(s) psicoactiva(s) junto con México en títulos, resúmenes y palabras clave ${ }^{5}$. El resto de los detalles se muestra a continuación (Tabla 1).

comprometían a prohibir la producción, distribución, venta y uso de las llamadas drogas.

${ }^{4}$ Los últimos tres instrumentos que miden las prevalencias nacionales $(2008,2011$ y 2016) han arrojado resultados al alza en cuanto al consumo de drogas alguna vez en la vida.

${ }^{5}$ No todas las bases de datos permiten la búsqueda en el cuerpo del texto. 
Tabla 1. Búsqueda de documentos y criterios de inclusión

\begin{tabular}{|c|c|c|c|c|c|}
\hline Base de datos & Scopus & Web of Science & PsycINFO & Science Direct & Redalyc \\
\hline Disciplinas & $\begin{array}{c}\text { Psychology, Social Sciences } \\
\text { y Multidisciplinary }\end{array}$ & $\begin{array}{c}\text { Health policy services, public } \\
\text { administration, law, } \\
\text { sociology, political science, } \\
\text { demography, geography, } \\
\text { social sciences (biomedical), } \\
\text { psychology } \\
\text { (multidisciplinary), sociale } \\
\text { sciences (interdisciplinary), } \\
\text { social issues, social work, } \\
\text { urban studies, psychology } \\
\text { (clinical), cultural studies, } \\
\text { psychology, anthropology, } \\
\text { history, social sciences } \\
\text { (mathematical methods) }\end{array}$ & $\begin{array}{l}\text { General (no tiene } \\
\text { opción para filtrar } \\
\text { por disciplina) }\end{array}$ & $\begin{array}{c}\text { General (no tiene } \\
\text { opción para filtrar } \\
\text { por disciplina) }\end{array}$ & $\begin{array}{c}\text { Psicología, } \\
\text { multidisciplinarias } \\
\text { (ciencias sociales), } \\
\text { sociología, política, } \\
\text { antropología, estudios } \\
\text { territoriales, filosofía, } \\
\text { derecho, estudios } \\
\text { culturales e historia }\end{array}$ \\
\hline $\begin{array}{l}\text { Fecha de } \\
\text { búsqueda }\end{array}$ & 11 de abril de 2018 & 20 de abril de 2018 & 12 de abril de 2018 & $\begin{array}{c}12-13 \text { de abril de } \\
2018\end{array}$ & 20 de abril de 2018 \\
\hline $\begin{array}{l}\text { Criterios de } \\
\text { inclusión }\end{array}$ & \multicolumn{5}{|c|}{$\begin{array}{l}\text { - Que el uso de drogas aparezca como tema central, o bien, que se trabaje con poblaciones usuarias de manera principal, } \\
\text { independientemente del tema. }\end{array}$} \\
\hline
\end{tabular}

Fuente: Elaboración propia

Se seleccionaron 155 documentos que se clasificaron a partir de sus poblaciones de estudio, lugares de investigación, drogas, metodologías, país de afiliación ${ }^{6}$ de las autoras y autores, y temáticas. Luego, se presentaron los resultados de la clasificación, donde se construyeron inductivamente una serie de categorías para cada clasificación. Después, se hizo un análisis de correspondencia entre las categorías temáticas y las demás clasificaciones, esto es, el ligue de las temáticas con las poblaciones de estudio, los estados del país, las drogas, las metodologías y los países de afiliación.

De esta manera, se busca conocer: las poblaciones, lugares, drogas y temáticas de las que se habla; las metodologías que más se utilizan y la afiliación de los autores y autora ${ }^{7}$; asimismo, conocer temáticas recurrentes en determinadas poblaciones y zonas del país, con determinadas drogas, metodologías y afiliaciones. Cabe señalar que, durante la revisión de los documentos, fue evidente que no todas las investigaciones versaban sobre una sola temática o

\footnotetext{
${ }^{6}$ Lugar de trabajo de investigadores e investigadoras, en el caso de trabajos con autoría múltiple, se tomó en cuenta la afiliación del autor o autora principal.

${ }^{7}$ Originalmente esta clasificación no estaba considerada, fue solo a partir de la revisión de los textos y el hecho de la existencia de muchas
}

trataban sobre un único grupo poblacional o una sola droga. Debido a ello, hubo documentos que se categorizaron con más de alguna temática, droga, población o lugar, por lo que los resultados dan cuenta de la ocurrencia con que alguna categoría fue encontrada en los documentos y no indican que hayan trabajado exclusivamente con alguna de ellas ${ }^{8}$.

Con esto, se busca conocer las tendencias en los estudios sobre drogas en México que, una vez discutidas con algunos elementos contextuales del país, servirán para plantear algunos vacíos y preguntas pertinentes que puedan servir de orientación para futuras investigaciones.

\section{RESULTADOS}

A continuación, se presentan de manera más detallada los resultados de cada una de las

investigaciones por parte de autores y autoras con afiliación a universidades e institutos fuera de México, que se consideró relevante la inclusión en el análisis.

${ }^{8}$ Con excepción de la clasificación por metodologías y país de afiliación, en estos casos las categorías fueron excluyentes. 
clasificaciones. Se inicia con las poblaciones, se continúa con lugares, drogas, metodologías, país de afiliación y, por último, las temáticas junto con su relación con las otras clasificaciones. Los porcentajes de inclusión de las categorías se sacaron en relación con los 155 documentos que integran este análisis.

\section{Poblaciones}

Con excepción de los trabajos enfocados en revisar el estado de conocimiento sobre algún tema relacionado con el uso de drogas, o aquellos cuyo interés estaba en analizar momentos históricos $y$, en menor medida, los que buscaban evaluar políticas; la mayoría de los textos trabajaron de alguna forma con una o varias poblaciones específicas (116 trabajos que representan el $74.83 \%$ de los 155 seleccionados).

La categorización de las poblaciones se hizo a partir del grupo etario al que pertenecían: niños (hasta 11 años); adolescentes (12 a 17 años); adultos (18 a 65 años); y adultos mayores (a partir de 66 años). A la par de estas categorías, construidas a partir de las características etarias de las poblaciones, fue muy recurrente la aparición de otros colectivos -caracterizados por los mismos autores y autoras- como los usuarios de drogas inyectables (UDI) ${ }^{9}$, las trabajadoras sexuales, estudiantes, migrantes y parejas de trabajadoras sexuales. La razón de incluir estas últimas categorías es que se considera relevante mostrar la regularidad con que otras cualidades o características de las personas fueron relacionadas con el estudio sobre drogas en México.

Tabla 2. Poblaciones

\begin{tabular}{lcc}
\hline Por edad & Cantidad & $\%$ \\
\hline Adultos & 95 & 61.29 \\
Adolescentes & 22 & 14.19 \\
Niños & 6 & 3.87 \\
Adultos mayores & 2 & 1.29 \\
\hline Otras poblaciones & Cantidad & $\%$ \\
\hline UDI & 48 & 30.96 \\
Trabajadoras sexuales (TS) & 23 & 14.83 \\
Estudiantes & 14 & 9.03 \\
Migrantes & 12 & 7.74 \\
Parejas de TS & 10 & 6.45 \\
\hline
\end{tabular}

Fuente: Elaboración propia.

\footnotetext{
${ }^{9}$ La población UDI se refiere a quienes se inyectan heroína, cocaína, metanfetamina o tranquilizantes. A diferencia de los usuarios de otras
}

Por un lado, en la Tabla 2 se aprecia el marcado interés que se tiene por la población adulta en las investigaciones sobre drogas en México, más de la mitad del total de los documentos analizados trabajaron con ella. Por otro lado, se observa una tendencia por estudiar a las poblaciones usuarias de drogas inyectables. Con menor interés, pero con prevalencias importantes, las investigaciones en adolescentes y trabajadoras sexuales.

\section{Lugares}

México cuenta con 32 entidades federativas conocidas como estados, de las cuales 19 tienen representación en este mapeo. De los 155 documentos analizados, 118 (el 76.12\%) versa sobre uno o más estados. Los restantes (23.87\%) tratan sobre política de drogas, el estado del conocimiento sobre algún tema relacionados con las drogas, o bien, no ligaban su investigación con algún estado en específico.

Tabla 3. Lugares de investigación

\begin{tabular}{|c|c|c|}
\hline Lugares & Cantidad & $\%$ \\
\hline Baja California & 62 & 40 \\
\hline CDMX & 34 & 21.94 \\
\hline Chihuahua & 20 & 12.90 \\
\hline Tamaulipas & 12 & 7.74 \\
\hline Jalisco & 9 & 5.81 \\
\hline Nuevo León & 4 & 2.58 \\
\hline Puebla & 3 & 1.94 \\
\hline Hidalgo & 2 & 1.29 \\
\hline Querétaro & 2 & 1.29 \\
\hline Campeche & 1 & 0.65 \\
\hline Chiapas & 1 & 0.63 \\
\hline Colima & 1 & 0.65 \\
\hline Estado de México & 1 & 0.65 \\
\hline Guanajuato & 1 & 0.65 \\
\hline Morelos & 1 & 0.65 \\
\hline Quintana Roo & 1 & 0.65 \\
\hline San Luis Potosí & 1 & 0.65 \\
\hline Sinaloa & 1 & 0.65 \\
\hline Yucatán & 1 & .065 \\
\hline
\end{tabular}

Fuente: Elaboración propia.

La Tabla 3 muestra una tendencia por el estudio de temáticas relacionadas con el uso de drogas en Baja California, específicamente en la ciudad de Tijuana, que es objeto de estudio en 59 de los 62 documentos sobre

drogas que no figuran en esta categorización, estos fueron incluidos por la regularidad con la que aparecieron. 
este estado. En las investigaciones sobre Baja California, destaca el interés en el estudio de colectivos como los usuarios de drogas inyectables, fuertemente ligados con poblaciones de trabajadoras sexuales y sus parejas, así como con población migrante y deportada ${ }^{10}$ de Estados Unidos. En general, la frontera norte de México se puede entender como un sitio de gran interés para las y los autores que trabajan con drogas, $4^{11}$ de los 6 estados con mayor prevalencia en el análisis son del norte de México y tienen frontera con los Estados Unidos.

Las otras 2 entidades federativas que se encuentran en los 6 primeros lugares son, la Ciudad de México -la capital del país y la zona metropolitana más poblada- y Jalisco, estado al que pertenece la ciudad de Guadalajara -segunda metrópoli más poblada de México-.

\section{Drogas}

El 41.29\% (64) de los documentos revisados para este trabajo aborda los temas relacionados con el uso de drogas de manera general, sin hacer referencia a ninguna en específico. El restante, 58.70\% (91) versa sobre una o más drogas o trabajaron con poblaciones usuarias de una o varias drogas. En la Tabla 4 se muestra la cantidad de investigaciones que incluyeron algunas de las drogas o grupos de drogas mencionadas.

Se observa un marcado interés por el estudio de las drogas inyectables $-29.03 \%$ del total de los trabajos considerados para este artículo-, sobre todo en la ciudad de Tijuana (Baja California) y en la frontera norte en general. De las investigaciones donde se aborda el estudio de estas drogas, 44 pertenecen a lugares de la frontera norte de México y solo 1 al resto del país (Ciudad de México). Todavía más, del total de documentos que se centran en una o más drogas, casi la mitad (49.45\%) incluyen en sus análisis a las drogas inyectables.

De manera individual, la marihuana es la droga que más aparece en los documentos, poco más del 10\% de los trabajos encontrados la incluyeron para estudiar: su relación con el suicidio; la comisión de delitos contra la propiedad; resultados de intervenciones; los argumentos pro y contra su legalización; y los factores asociados a su

\footnotetext{
${ }^{10}$ La ciudad de Tijuana, en el norte de México, tiene frontera con el estado de California en Estados Unidos.

${ }^{11}$ Baja California, Chihuahua, Tamaulipas y Nuevo León.

${ }^{12} \mathrm{Si}$ bien, al hacer referencia a drogas inyectables no se habla sino de varias drogas (heroína, metanfetaminas, cocaína y tranquilizantes), se
}

uso en adolescentes. La mitad de los trabajos (8) que tratan sobre marihuana están relacionados con poblaciones de adolescentes en contextos escolares, lo que da cuenta del interés que tiene esta población en relación con esta droga. Después de la marihuana, aparece el crack con 10 trabajos que representan el 6.45\% del total de las investigaciones. Los temas que destacan tienen que ver la evaluación de intervenciones (4), los patrones de uso y el perfil de los usuarios (4), asimismo con los riesgos y comportamientos asociados a su uso (3). Destacan, también, las poblaciones de adultos (8), las trabajadoras sexuales (3) y sus parejas (3).

Tabla 4. Drogas

\begin{tabular}{|c|c|c|}
\hline Drogas & Cantidad & $\%$ \\
\hline $\begin{array}{l}\text { Drogas } \\
\text { inyectables }{ }^{12}\end{array}$ & 45 & 29.03 \\
\hline Marihuana & 16 & 10.32 \\
\hline Crack & 10 & 6.45 \\
\hline Cocaína & 9 & 5.80 \\
\hline Metanfetaminas & 7 & 4.51 \\
\hline Inhalantes & 7 & 4.51 \\
\hline Heroína & 5 & 3.22 \\
\hline Peyote & 5 & 3.22 \\
\hline $\begin{array}{l}\text { Hongos } \\
\text { psilocibios }\end{array}$ & 2 & 1.29 \\
\hline Opio & 2 & 1.29 \\
\hline Ayahuasca & 1 & 0.64 \\
\hline DMT (Sapo) & 1 & 0.64 \\
\hline Éxtasis & 1 & 0.64 \\
\hline Tranquilizantes & 1 & 0.64 \\
\hline Poppers & 1 & 0.64 \\
\hline Datura & 1 & 0.64 \\
\hline Olioliuqui & 1 & 0.64 \\
\hline
\end{tabular}

Fuente: Elaboración propia.

La cocaína apareció en 9 de los textos revisados (5.80\%). En general, destacan temas como los riesgos y comportamientos asociados al uso de drogas (4),

decidió incluir este término debido al alto porcentaje de documentos que hacían referencia al mismo. Cuando las drogas que vienen incluidas en este término aparecieron de forma individual, no se sumaron a esta categoría porque no necesariamente hacen referencia a esta vía de administración. 
patrones y perfil de usuarios (4), el ambiente cultural que rodea a su uso (2) y la evaluación de intervenciones con usuarios de esta droga (s). En particular, aparecen temas como la relación del uso de cocaína en el sueño y en la ideación suicida, en la producción cultutal y en el significado asignado por estudiantes. La población adulta fue la más estudiada (5), seguida de los grupos de adolescentes, estudiantes, trabajadoras sexuales y sus parejas (2). La metanfetamina apareció en 7 documentos (4.51\%). Los temas que trataron sobre ésta fueron el ambiente cultural (2), los riesgos y comportamientos asociados al uso de drogas (2), el patrón de uso y perfil de usuario (2), los factores de protección y de riesgo (2), la evaluación de intervenciones (2) y la perspectiva de género (1). Particularmente, destacan temáticas como los factores que inciden en los diferentes géneros para al inicio y mantenimiento en el uso de metanfetaminas. Las poblaciones que fueron mayormente estudiadas fueron los adultos (5) y, menormente, los adolescentes (i). También, aparecen trabajadoras sexuales (4), sus parejas (3) y grupos de estudiantes (1).

El uso de solventes inhalantes apareció en 7 trabajos (4.51\%). El ambiente cultural fue el tema más estudiado en estas investigaciones (4), seguido del patron de uso y perfil de usuario (2), luego, riesgos y comportamiento asociados al uso de drogas, la evaluación de intervenciones y la revisión bibliográfica con 1 trabajo cada uno. Particularmente, fue relevante el estudio etnográfico en niños en condición de calle de la Ciudad de México, siendo estudiado los significados, la importancia del consumo para una diversidad de personas y la agencia de los usuarios. Las poblaciones más estudiadas fueron adolescentes y niños con 4 trabajos cada una, mientras que los adultos emergieron en 2 ocasiones.

El peyote apareció en 5 de los trabajos analizados (3.22). Fue abordado para la evaluación de políticas (3), el análisis del ambiente cultural (2), en investigaciones históricas (2), para encontrar riesgos y comportamientos asociados a su uso (1), y para evaluar intervenciones (1). Fue relevante la discusión sobre su estatus legal, su uso en contextos ceremoniales y los argumentos para su reconocimiento como patrimonio biocultural del país, así como su posible legalización para su uso en personas no pertenecientes a pueblos indígenas.

\section{Temáticas de los documentos}

Se construyeron nueve categorías temáticas, de manera inductiva, con el objetivo de hacer visibles los temas de los que se habla en el corpus de datos utilizados. Es necesario recordar que las investigaciones pueden tratar de uno o más temas, dependiendo de sus objetivos.

Tabla 5. Temáticas de los documentos

\begin{tabular}{|c|c|c|}
\hline Tema & Cantidad & $\%$ \\
\hline $\begin{array}{l}\text { Evaluación y análisis de } \\
\text { intervenciones y } \\
\text { procesos de } \\
\text { rehabilitación }\end{array}$ & 36 & 23.22 \\
\hline Evaluación de políticas & 34 & 21.93 \\
\hline $\begin{array}{l}\text { Riesgos y } \\
\text { comportamientos } \\
\text { asociados al uso de } \\
\text { drogas }\end{array}$ & 34 & 21.93 \\
\hline $\begin{array}{l}\text { Factores de protección y } \\
\text { de riesgo }\end{array}$ & 29 & 18.70 \\
\hline $\begin{array}{l}\text { Patrón de uso y perfil de } \\
\text { usuario }\end{array}$ & 28 & 18.06 \\
\hline Ambiente cultural & 20 & 12.90 \\
\hline Investigaciones históricas & 6 & 3.87 \\
\hline Uso de drogas y género & 6 & 3.87 \\
\hline Estados de la cuestión & 3 & 1.93 \\
\hline
\end{tabular}

Fuente: Elaboración propia

A continuación se presenta una descripción de las categorías temáticas y su relación con las poblaciones, las entidades federativas y las drogas encontradas en los documentos.

Evaluación y análisis de intervenciones y procesos de rehabilitación

Trabajos que analizan los impactos de las intervenciones sobre dependencia a drogas, a corto, mediano o largo plazo. Entre los principales impactos que estudian están: los efectos de los tratamientos involuntarios sobre la ocurrencia de sobredosis; evaluaciones del riesgo de que usuarios inicien a otros en el consumo de drogas; la identificación de municipios con prioridad de servicios; evaluaciones sobre la aceptabilidad de los programas en las personas usuarias. Asimismo, estos trabajos muestran gran interés por las barreras existentes en cuanto al acceso a procesos de 
intervención: van desde los análisis de las características de los usuarios que presentan menor o mayor disposición a los tratamientos; hasta las barreras estructurales, por parte de gobiernos o policías, que se manifiestan en procesos de índole burocrática, en la confiscación de objetos destinados para el uso de drogas, extorsiones y violencia.

Además, se hallan los análisis de los procesos de rehabilitación, desde la situación social de los internos, los procesos narrativo-conversacionales y la importancia de las metáforas en determinadas intervenciones, hasta el papel de la masculinidad hegemónica en estos centros de tratamiento. Las poblaciones y drogas que más se estudian son los adultos (22) y las drogas inyectables (8); las entidades federativas donde se concentró el interés por esta temática fueron la Ciudad de México (12) y Baja California (11).

\section{Evaluación de políticas}

La evaluación de los resultados de las políticas de drogas, de reformas a leyes de seguridad y salud o las barreras o incentivos para la correcta implementación de éstas son algunos de los principales temas tratados dentro de los trabajos incluidos en esta categoría. Específicamente, los temas van desde los daños sociales y económicos producidos por la guerra contra las drogas en México, la exposición de argumentos para la legalización de la marihuana, la exposición de los contras de esta posibilidad, e incluso, sobre cómo el derecho a la salud se ve coartado por la política de drogas. También, son muy recurrentes los documentos que evalúan desde perspectivas de salud pública, el papel de las policías en el contexto de reforma a la Ley del Narcomenudeo del $2009^{13}$. Dichos trabajos muestran que las policías siguen deteniendo y extorsionando a portadores de drogas sin ninguna canalización a autoridades sanitarias.

El vínculo de esta temática se da principalmente con poblaciones adultas (12), con drogas inyectables (12). Respecto a los lugares, y en tanto se evalúan políticas de drogas, las investigaciones se centran en hablar del país en general (17). No obstante, hay trabajos que se dedican a evaluar dichas políticas en la práctica: es el caso de los

\footnotetext{
${ }^{13}$ En esta reforma se estipularon cantidades máximas de portación para uso personal y que quienes fueran sorprendidos con dichas cantidades se canalizarían a autoridades de salud.
}

interesados por la aplicación de la reforma a la Ley del Narcomenudeo 2009 en el estado de Baja California (16).

Riesgos y comportamientos asociados al uso de drogas

La mayor parte de los trabajos de esta categoría son del campo de la epidemiología y la salud pública, pueden dividirse entre los que buscan riesgos relacionados con el uso de drogas y aquellos que buscan conocer qué conductas y circunstancias lo acompañan. Los primeros buscan consecuencias físicas del uso de drogas como el riesgo de abortos en poblaciones usuarias, los efectos de determinadas drogas en el sueño de las personas, las consecuencias del empleo de determinados objetos para el uso de drogas y generalmente utilizan poblaciones de referencia para comparar una o más variables con los grupos de usuarios que interesa observar.

Los segundos, buscan otro tipo de correlaciones que no son necesariamente físicas, por ejemplo: conocer qué variables del cuidado a la salud acompañan a los usos de drogas; medir la comisión de crímenes contra la propiedad en usuarios de marihuana; conocer la relación entre el uso de drogas y la ideación suicida; las circunstancias que acompañan a los usuarios de drogas inyectables en condición de migrantes; las características demográficas de determinadas poblaciones de usuarios, etc.

Estos trabajos están principalmente relacionados con poblaciones adultas (26), entre las que destacan los grupos de usuarios de drogas inyectables (19) y las trabajadoras sexuales (12). Las entidades federativas relacionadas mayoritariamente con esta categoría son Baja California (21) y Chihuahua (12), ambas del norte de México y con frontera con Estados Unidos.

\section{Factores de protección y de riesgo}

Esta categoría se construyó a partir de trabajos que abordan los factores que pueden incidir o evitar el uso de diferentes drogas; y a diferencia de la categoría anterior, donde se miden riesgos y buscan correlaciones desde la epidemiología, los factores de protección y de riesgo son mayormente desarrollados desde la psicología. Incluyen factores como los contextos familiares: la comunicación y la supervisión por parte de los padres, las adversidades 
familiares en la infancia, el haber sido abusado o no durante la infancia; los contextos sociales: la disponibilidad de drogas, el nivel de urbanización de los lugares de residencia, el tener amigos usuarios o no usuarios de drogas, con conductas antisociales o no, la tolerancia al uso en ciertos contextos; contextos escolares: las expectativas académicas, la migración interna por ingreso a la educación superior; las cuestiones subjetivas: las actitudes hacia el uso de drogas, las percepciones, los significados asignados a las drogas, el control conductual, los roles, el amor en las relaciones de pareja, etc.

La población adulta es el objeto del mayor interés (22), no obstante, vale la pena señalar que esta categoría es donde más investigaciones en adolescentes se encuentran (8), mayoritariamente realizadas en contextos estudiantiles (6), dejando claro el interés que se tiene por la iniciación en el uso de drogas. Asimismo, se observa una relación importante con las y los usuarios de drogas inyectables (9) y los migrantes (7). La categoría suele ser abordada de manera general en cuanto al uso de drogas se refiere (14), con excepción de los trabajos sobre drogas inyectables (9) y se concentra en los estados de Baja California (10) y Tamaulipas (7), ambos del norte de México.

\section{Patrón de uso y perfil de usuario}

Incluye investigaciones que buscan indagar sobre la prevalencia, frecuencia, duración, distribución y demás dinámicas que acompañan al uso de cualquier droga, como las pautas de iniciación y progresión de los usos. Se analizan desde formas de intoxicación encontradas en productos culturales como la literatura, pasando por instrumentos aplicados por autoridades de salud, y hasta los resultados de trabajos etnográficos. De esta forma, se suelen definir perfiles de usuarios de diferentes drogas, utilizando diferencias de género, sociodemográficas, etcétera. Asimismo, se hacen perfiles de aquellos usuarios que buscan cesar el consumo. La categoría se concentra en poblaciones adultas (22) y de usuarios de drogas inyectables (9), en el estado de Baja California (14) y en la Ciudad de México (8).

\section{Ambiente cultural}

Esta categoría se puede dividir en dos aspectos esenciales, por un lado, el análisis de las maneras en que la sociedad entiende el uso de drogas: por medio de analizar discursos, imaginarios sociales, productos culturales, modelos de entendimiento, actitudes hacia las políticas de drogas y las consecuencias que esto tiene para los usuarios, dígase del estigma, la discriminación, etcétera. Por otro lado, los aspectos que tienen que ver con la perspectiva de los usuarios: trabajos distanciados del énfasis en las consecuencias negativas de los usos, estudiando percepciones, significados, semántica y prácticas socialmente significativas y cargadas de agencia en lo que al uso de drogas se refiere.

Los adultos son los más estudiados (8), aunque se aprecia cierto interés por adolescentes (4) y niños (4), destacando los trabajos sobre los significados asignados por ellos al concepto de droga y aquellos sobre el uso de inhalantes en la Ciudad de México. Destaca también el interés por analizar el papel que juegan las relaciones amorosas entre trabajadoras sexuales y sus compañeros en contextos de uso de drogas inyectables. Las drogas más comunes en esta categoría fueron las investigaciones sobre inhalantes (4) y las drogas inyectables (4), y las entidades federativas fueron la Ciudad de México (7) y Baja California (7).

\section{Investigaciones históricas}

Construida a partir de documentos que abordan la historia de las prohibiciones y regulaciones de drogas como el opio (2), el peyote (2) y la marihuana (1). Buscan tener una comprensión de los discursos históricos oficiales y del contexto sociocultural, por medio de metodologías cualitativas y través de la revisión de periódicos, documentos oficiales, leyes, entrevistas, etc. Esta categoría no tiene ninguna relación con poblaciones específicas y solo aparece el estado de Baja california y Jalisco, en una ocasión cada uno, para discutir la regulación del peyote y los discursos del Estado mexicano al respecto.

\section{Uso de drogas y género}

Las investigaciones donde se discuten estas temáticas abordan el papel del género en el uso de drogas, partiendo de las diferentes "rutas" de iniciación entre hombres y mujeres, pasando por diferentes maneras de uso estable e incluso diferencias en las formas de acceder a los contextos de rehabilitación. Sobre las mujeres, los estudios tienen que ver con drogas de alto impacto como el crack y las metanfetaminas, argumentan que la iniciación en éstas se da, muchas 
veces, por influencia de parejas masculinas y que al momento de acceder a tratamientos tienen más barreras que los hombres.

Sobre los hombres, señalan el papel que juega la masculinidad hegemónica en la iniciación, uso regular y al interior de los centros de rehabilitación -lugares donde además se reproduce la violencia ligada a la masculinidad hegemónica-. Se argumenta que el uso de drogas de alto impacto tiene que ver con el poder, como una forma de asumir el control, como un dictamen de la masculinidad hegemónica de enrolarse en actividades de riesgo para tener reconocimiento en determinados contextos. Esta categoría se concentra en adultos (6), en la Ciudad de México (4) y el uso de drogas aparece de manera general.

\section{Revisiones bibliográficas}

Los estudios encontrados para esta categoría fueron únicamente 2 -sin relación con ninguna población o lugar del país. Se centran en identificar el estado del conocimiento sobre drogas como los solventes inhalables (1) y sus alternativas de prevención, así como conocer los tratamientos y posibles usos terapéuticos de la marihuana (1).

\section{Metodologías}

Los resultados acerca de las metodologías usadas en las investigaciones analizadas muestran que la metodología cuantitativa es la que mayoritariamente se emplea, con un 59.35\% de los de documentos; los métodos cualitativos suman un 34.19\%; y los métodos mixtos tan solo un $6.45 \%$. La mayor parte de los estudios cuantitativos utiliza técnicas de la salud pública para relacionar usos con determinados riesgos 0 comportamientos (28), para encontrar factores que incentiven o prevengan esos usos (23), para medir prevalencias y patrones de uso (22), así como para evaluar el éxito de programas de intervención (18), y valorar la puesta en práctica de reformas a leyes (13) principalmente la reforma a la Ley de Narcomenudeo 2009-.

Aquellas investigaciones que emplean metodologías cualitativas tratan, principalmente, sobre análisis teóricos de políticas de drogas (18), sobre el ambiente cultural que rodea al uso drogas (16) -percepciones, significados, prácticas-, y sobre los contextos de intervención (14) -la situación social de los enfermos y el papel de las narrativas en dichas intervenciones-.

\section{País de afiliación de los autores}

La afiliación de los autores de los documentos hace referencia al país donde está la universidad o instituto donde desarrollan su labor investigativa, para el caso de los documentos con dos o más autores, se tomó como país de afiliación el lugar correspondiente del que aparece como autor principal. La afiliación principal de las y los autores fue estadounidense, con 84 trabajos que representan el 54.19\% del total de las investigaciones. Después vienen las y los autores de México, con 64 trabajos que representan el $41.29 \%$ del total de documentos. Por último, 7 trabajos repartidos entre autores de Brasil, Países Bajos, Chile y la India.

El $76.19 \%$ de las investigaciones estadounidenses fueron realizadas en la frontera norte de México. Los temas sobre los que versan son en su mayoría relacionados a los campos de la salud pública y por medio de metodologías cuantitativas: riesgos y comportamientos asociados al uso de drogas; evaluación de intervenciones; patrón y perfil de usuario; y evaluación de políticas. Las poblaciones mayormente incluidas son las trabajadoras sexuales (23) y sus parejas (10). Las drogas más estudiadas son las inyectables (43) y el crack (8)

Por su lado, en las investigaciones mexicanas los temas más estudiados fueron: la evaluación de políticas de drogas, abordada de manera cualitativa sobre el país en general -a diferencia de las y los autores estadounidenses que mayoritariamente se dedicaron a valorar la puesta en práctica de leyes en la frontera norte; los factores de protección y de riesgo; y la evaluación de intervenciones. Los lugares más frecuentes fueron la Ciudad de México (25) y el estado de Tamaulipas (7). Las drogas más estudiadas fueron la marihuana (8) y la cocaína (5). Y las poblaciones más recurrentes fueron los estudiantes (9) y los migrantes (3).

\section{DISCUSIÓN}

Los resultados del análisis descriptivo muestran una tendencia en cuanto a abordar el uso de drogas por medio de análisis empíricos, casi tres cuartas partes de 
los trabajos analizados (74.83\%) trabajaron con algún tipo de población. Esto puede ser el reflejo de la predominancia de trabajos que tratan de temas que generalmente se abordan desde la epidemiología y en menor medida desde la psicología: aquellos que buscan riesgos y comportamientos asociados al uso de drogas; tasas de prevalencia y patrones de consumo; el entorno personal como factor protección o de riesgo; y la eficacia de las intervenciones. Estos temas generalmente son abordados con técnicas de seguimiento y metodologías cuantitativas del campo de salud pública (París y Pérez, 2012) y trabajan con poblaciones consideradas en riesgo de uso de drogas.

Se encontró que, de los 155 trabajos más de la mitad incluyó el estudio de población adulta (61.29\%), mientras que, si se toma en cuenta únicamente a los que utilizaron alguna muestra, se tiene que la mayoría (79.89\%) incluyeron adultos. La ENCODAT 2016-2017 ${ }^{14}$ (2017) señala que la mayor parte del uso de drogas alguna vez en la vida se concentra en la población de 18 a 65 años (15\%), por encima de la población de 12 a 17 años (6.4\%). Asimismo, el elevado interés por los adultos puede deberse a que la población económicamente activa es la que sufre de la mayor parte de los problemas de salud relacionados con el uso de drogas (López et al, 2006, citado en Bojórquez y Cortés, 2012), lo que tiene un gran impacto sobre el desarrollo de los países (Bojórquez y Cortés, 2012).

Existen estudios que señalan que el uso de drogas se presenta igualmente en todos los estratos socioeconómicos (Mosher y Yanagisako, 1991, citado en París, 2012). Asimismo, la ENCODAT 2016-2017 (2017) señala que el uso de heroína alguna vez en la vida tiene un porcentaje muy bajo (0.2\%) y el informe del Sistema de Vigilancia Epidemiológica de las Adicciones 2016 (SISVEA) (2016) apunta que la heroína es la sexta droga de impacto ${ }^{15}$ después del alcohol, cristal (metanfetaminas), marihuana, cocaína e inhalantes.

A pesar de lo anterior, son mayoría las investigaciones que tratan sobre drogas inyectables (48 investigaciones) y trabajadoras sexuales (23 investigaciones), en su mayoría estudiadas en la Ciudad de Tijuana en el estado de Baja California por parte de

\footnotetext{
${ }^{14}$ La población objetivo de esta encuesta son las personas de 12 a 65 años y sus cuestionarios los dividen entre poblaciones menores y mayores de edad.
}

autores afiliados a Estados Unidos. Con esto, se advierte que existe un excesivo interés por estos grupos y drogas, con lo que no se trata de negar el impacto que tienen las drogas inyectables en las personas, simplemente se argumenta que este interés no se corresponde con la situación del contexto mexicano y que además parece coadyuvar con la discriminación y el estigma hacia ciertos colectivos, sugiriendo que el uso de drogas se reduce a ciertos grupos problematizados.

Sin embargo, si se toma en cuenta únicamente a los estudios realizados por autores con afiliación mexicana tenemos que las drogas y grupos más estudiados son la marihuana y la cocaína, junto con estudiantes y migrantes. Estos estudios parecen tener una mayor correspondencia con el contexto mexicano, pues las drogas con mayor prevalencia de uso en el país son precisamente esas dos (Comisión Nacional Contra las Adicciones-Instituto Nacional de Psiquiatría Ramón de la Fuente Muñiz, 2017). Mientras que, del lado de las poblaciones, el interés por estudiantes puede deberse a que de acuerdo con la ENCODAT 2017-2017 (2017) el uso de cualquier droga alguna vez en la vida por parte de poblaciones de 12 a 17 casi se dobló en el 2016 (6.4\%) en comparación con el instrumento del 2011 (3.3\%). Por lo tanto, dicho interés parece estar justificado en dichos datos y se puede interpretar como un esfuerzo por reducir dicha tendencia.

El que la mayoría de las investigaciones hayan sido realizadas en las entidades federativas que tienen frontera con los Estados Unidos puede apuntar, por un lado, a una correspondencia con datos que indican que el consumo de drogas es mayor en esa región (Comisión Nacional Contra las Adicciones-Instituto Nacional de Psiquiatría Ramón de la Fuente Muñiz, 2017). Por otro, que es consecuencia de la gran cantidad de investigaciones por parte de autores afiliados a universidades y centros de investigación de los Estados Unidos. Que en su mayoría (76.19\%), fueron realizadas en las ciudades fronterizas del norte de México. Ahora bien, tomando en cuenta a los autores con afiliación mexicana, tenemos que la mayoría investiga sobre la Ciudad de México (25), que está apenas por encima (10.6\%) de la media nacional (10.3\%) en cuanto a consumo de drogas alguna vez en la vida, por detrás de más de diez entidades

\footnotetext{
15 En el informe del SISVEA, el término de "droga de impacto" hace referencia a las drogas que motivan ingresos en centros de rehabilitación no gubernamentales.
} 
federativas, entre las que destacan el estado de Quintana Roo que, a pesar de tener la segunda prevalencia más alta de uso alguna vez en la vida (14.9\%), únicamente apareció en una de 155 investigaciones.

En lo que respecta a las temáticas abordadas en las investigaciones se encontró una tendencia por abordar los temas que generalmente se abordan desde la epidemiología, sobre todo en las investigaciones afiliación estadounidense. Mientras que, en las investigaciones mexicanas, se encontró un énfasis por la evaluación de la política de drogas desde una perspectiva crítica y después por los factores que pueden incidir o proteger en el uso de drogas. Sobre estos resultados, puede decirse que como señala Pérez Montfort (2016), las investigaciones sobre drogas siguen reproduciendo el esquema prohibicionista. La poca aparición de temas que aborden a los usuarios como sujetos de derecho y la nula aparición de usuarios 'normalizados' ${ }^{16}$ fuera de colectivos considerados en riesgo de uso, muestra que el uso de drogas sigue entendiéndose como parte de sectores exclusivos, lo que a su vez contribuye a la estigmatización y discriminación de estos.

Para finalizar, es importante apuntar a la falta y la necesidad de investigaciones desde una perspectiva que parta de la diversidad humana, del entendimiento de que no todos los usos devienen necesariamente problemáticos, de la significancia del uso para ciertas personas y de la agencia que tienen para decidir usar drogas. Incluso, vale argumentar que ante la inminente legalización de la marihuana son necesarias investigaciones que aborden, como bien señala De la Fuente (2015), el significado que tiene el uso de esta planta en el México contemporáneo, de una forma que se aleje de una conceptualización desde la marginalidad y la delincuencia y que permita conocer cómo los usuarios interpretan y reinterpretan los símbolos asociados con el uso de ésta. Asimismo, vale la pena preguntarse por la manera en que los usuarios que, en su afán por permanecer institucionalizados, gestionan tanto sus usos como el estigma proveniente del contexto social.

\footnotetext{
${ }^{16}$ Entendidos como aquellos que permanecen institucionalizados, que no significan el uso de drogas como una forma de resistencia a poderes establecidos.
}

\section{Agradecimientos}

Este trabajo ha sido realizado en el marco del programa de doctorado en Persona y Sociedad en el Mundo Contemporáneo de la Universidad Autónoma de Barcelona.

\section{REFERENCIAS}

Comisión Nacional Contra las Adicciones-Instituto Nacional de Psiquiatría Ramón de la Fuente Muñiz. (2017). Encuesta Nacional de Consumo de Drogas, Alcohol y Tabaco 2016-2017. Ciudad de México: Instituto Nacional de Psiquiatría Ramón de la Fuente Muñiz-Secretaría de Salud.

De la Fuente, R. (2015). Marihuana y salud. México, DF: FCE-UNAM-ANM.

Dirección General de Epidemiología. (2016). Informe SISVEA 2016. México: Secretaría de Salud.

Expansión. (02 de julio de 2018). Este es el discurso completo que dio AMLO en el Zócalo. Expansión. Recuperado de https://expansion.mx/nacional/2018/07/02/este-es-discursocompleto-que-dio-amlo-en-el-zocalo.

Miranda, J. (21 de agosto de 2018). Alfonso Durazo se pronuncia a favor de la legalización de la marihuana. El Universal. Recuperado de http://www.eluniversal.com.mx/nacion/politica/alfonso-durazo-sepronuncia-favor-de-la-legalizacion-de-la-marihuana

París Pombo, M. D. (2013). Criminalización del consumo de drogas y derechos humanos de los usuarios. El caso de Tijuana, Baja California. En María Dolores, París Pombo y Lorena Raquel, Pérez Floriano (Eds.), La marca de las drogas: Violencias y prácticas de consumo (pp. 109-136). Tijuana: El Colegio de la Frontera Norte-Juan Pablos Editor.

Pérez, R. (2016). Tolerancia y prohibición. Aproximaciones a la historia social y cultural de las drogas en México 1840-1940. México, D.F.: Debate.

Reséndiz, F. (22 de agosto de 2018). Gobierno de AMLO planteará a ONU despenalización de drogas. El Universal. Recuperado de http://www.eluniversal.com.mx/nacion/politica/gobierno-de-amloplanteara-onu-despenalizacion-de-drogas-sanchez-cordero

Secretariado Ejecutivo del Sistema Nacional de Seguridad Pública. (2012). Incidencia Delictiva del Fuero Común 2006. México: Centro Nacional de Información.

Secretariado Ejecutivo del Sistema Nacional de Seguridad Pública. (2018). Incidencia Delictiva del Fuero Común 2017. México: Centro Nacional de Información.

Suprema Corte de Justicia de la Nación. (4 de julio de 2018). Comunicados. Ciudad de México. Suprema Corte de Justicia de la Nación. 
Recuperado

de http://www.internet2.scjn.gob.mx/red2/comunicados/noticia.asp?i $\underline{d=4738}$

\section{Referencias del análisis}

Aguilar Bustos, O. E., Valdez Gonzales, G. R., Brambila, M. A., Gallegos Cari, A., Camacho Solís, R., \& Mendoza Meléndez, M. A. (2013). Problematic consumption of drugs and risk or protection factors in a reclusion center of the city of Mexico. International Journal of Psychological Research, 6(1), 78-83.

Andrade Palos, P., Betancourt-Ocampo, D., Moreno-Carmona, N. D., \& Alvis-Rizzo, A. (2017). Fortalezas externas desde el modelo de desarrollo positivo de los jóvenes y consumo de sustancias en una muestra de adolescentes mexicanos y colombianos. Avances en Psicología Latinoamericana, 35(3), 515-529. doi: $\underline{10.12804 / \text { revistas.urosario.edu.co/apl/a.4095 }}$

Arellanez Hernández, J. L. (2016). Consumo de drogas y respuestas al estrés migratorio entre los migrantes mexicanos que se dirigen a Estados Unidos. Frontera Norte, 28(56), 113-133.

Arredondo, J., Gaines, T., Manian, S., Vilalta, C., Bañuelos, A., Strathdee, S. A., \& Beletsky, L. (2018). The law on the streets: Evaluating the impact of Mexico's drug decriminalization reform on drug possession arrests in Tijuana, Mexico. International Journal of Drug Policy, 54, 1-8. doi: 10.1016/j.drugpo.2017.12.006

Arredondo, J., Strathdee, S. A., Cepeda, J., Abramovitz, D., Artamonova, I., Clairgue, E., ... Beletsky, L. (2017). Measuring improvement in knowledge of drug policy reforms following a police education program in Tijuana, Mexico. Harm Reduction Journal, 14. doi: $\underline{10.1186 / s 12954-017-0198-2}$

Arroyo, M., Natera, G., Gelberg, L., Andersen, R., \& Rico, M. (2015). Prevalence and correlates of drug use in women patients of community health centers in Tijuana, Mexico and Los Angeles, USA. Drug and Alcohol Dependence, 156, e10-e11. doi: 10.1016/j.drugalcdep.2015.07.947

Bazzi, A. R., Syvertsen, J. L., Luisa Rolon, M., Martinez, G., Rangel, G., Vera, A., ... Strathdee, S. A. (2016). Social and Structural Challenges to Drug Cessation Among Couples in Northern Mexico: Implications for Drug Treatment in Underserved Communities. Journal of Substance Abuse Treatment, 61, 26-33. doi: 10.1016/j.jsat.2015.08.007

Beletsky, L., Lozada, R., Gaines, T., Abramovitz, D., Staines, H., Vera, A., ... Strathdee, S. A. (2013). Syringe confiscation as an HIV risk factor: The public health implications of arbitrary policing in Tijuana and Ciudad Juarez, Mexico. Journal of Urban Health, 90(2), 284-298. doi: $\underline{10.1007 / s 11524-012-9741-3}$

Beletsky, Leo, Wagner, K. D., Arredondo, J., Palinkas, L., Magis Rodriguez, C., Kalic, N., ... Strathdee, S. A. (2016). Implementing Mexico's «Narcomenudeo» Drug Law Reform: A Mixed Methods Assessment of Early Experiences Among People Who Inject Drugs. Journal of Mixed Methods Research, 10(4), 384-401. doi: $\underline{10.1177 / 1558689815575862}$

Ben Hamida, A., Rafful, C., Jain, S., Sun, S., Gonzalez-Zuniga, P., Rangel, G., ... Werb, D. (2018). Non-injection Drug Use and Injection Initiation
Assistance among People Who Inject Drugs in Tijuana, Mexico. Journal of Urban Health, 95(1), 83-90. doi: 10.1007/s11524-017-01884

Benjet, C., Borges, G., Medina-Mora, M. E., \& Méndez, E. (2013). Chronic childhood adversity and stages of substance use involvement in adolescents. Drug and Alcohol Dependence, 131(1-2), 85-91. doi: 10.1016/j.drugalcdep.2012.12.002

Bermúdez Lozano, P., Medina-Mora Icaza, M. E., Berenzon Gorn, S., García Cabrero, B., Amador Buenabad, N., \& Pérez López, C. (2014). Significado psicológico que estudiantes de secundaria de la Ciudad de México atribuyen al concepto "drogas". Revista Intercontinental de Psicología y Educación, 16(1), 11-36.

Booth, J. M., Marsiglia, F. F., Nuno-Gutierrez, B. L., \& Garcia Perez, M. (2014). The Association between Engaging in Romantic Relationships and Mexican Adolescent Substance Use Offers: Exploring Gender Differences. Substance Use \& Misuse, 49(11), 14801490. doi: $10.3109 / 10826084.2014 .913627$

Borges, G., Benjet, C., Orozco, R., Medina-Mora, M.-E., \& Menendez, D. (2017). Alcohol, cannabis and other drugs and subsequent suicide ideation and attempt among young Mexicans. Journal of Psychiatric Research, 91, 74-82. doi: 10.1016/j.jpsychires.2017.02.025

Borges, G., Cherpitel, C. J., Orozco, R., Zemore, S. E., Wallisch, L., MedinaMora, M.-E., \& Breslau, J. (2016). Substance use and cumulative exposure to American society: Findings from both sides of the US Mexico border region. American Journal of Public Health, 106(1), 119. 127. doi: 10.2105/AJPH.2015.302871

Campos, I. (2012). Home Grown: Marijuana and the Origins of Mexico's War on Drugs (Vol. 41). Chapel Hill: The University of North Carolina Press.

Capo Valdivia, Z. V. (2015). Clandestinity, taxation and tolerance: Opium in the official discourse, Mexicali, Mexico 1915-1916. Culturales, 3(2), 77-111.

Castro, A. O., García, M. J. D., Calderón, G. P., \& Medina-Mora, M. E. (2017). Activo distribution and paraphernalia among «street children». Salud Mental, 40(4), 165-170. doi: 10.17711/SM.0185-3325.2017.021

Cepeda, A., Nowotny, K. M., \& Valdez, A. (2015). Injecting drug use among Mexican Female Sex Workers on the US-Mexico Border. Journal of Ethnicity in Substance Abuse, 14(4), 351-363. doi: $\underline{10.1080 / 15332640.2014 .991467}$

Cepeda, Alice, Frankeberger, J., Bailey, J. L., Nowotny, K. M., Natera-Rey, G., \& Valdez, A. (2017). HIV and STI knowledge, testing, and risk among adult crack users in Mexico city: baseline findings from a feasibility study. Aids Care-Psychological and Socio-Medical Aspects of Aids/Hiv, 29(3), 350-354. doi: 10.1080/09540121.2016.1255707

Cepeda, J. A., Strathdee, S. A., Arredondo, J., Mittal, M. L., Rocha, T., Morales, M., ... Beletsky, L. (2017). Assessing police officers' attitudes and legal knowledge on behaviors that impact HIV transmission among people who inject drugs. International Journal of Drug Policy, 50, 56-63. doi: 10.1016/j.drugpo.2017.09.009 
Chavez Guzmán, G. M. (2017). Interculturalidad en torno al uso del peyote. Un patrimonio biocultural en condición de ilegalidad. Alteridades, 27(53), 95-106.

Collins, K. M. (2016). Mobile Technology Use Among Persons who Inject Drugs in San Diego, California and Tijuana, Baja California, Mexico. UC San Diego, US.

Conners, E. E., Gaines, T. L., Strathdee, S. A., Magis-Rodriguez, C., \& Brouwer, K. C. (2017). Structural factors associated with methamphetamine smoking among female sex workers in tijuana, mexico. Drug and Alcohol Review, No Pagination Specified-No Pagination Specified. doi: 10.1111/dar.12633

Conners, E. E., Swanson, K., Morales-Miranda, S., Fernandez Casanueva, C., Mercer, V. J., \& Brouwer, K. C. (2017). HIV Risk Behaviors and Correlates of Inconsistent Condom Use Among Substance Using Migrants at the Mexico/Guatemala Border. Aids and Behavior, 21(7), 2033-2045. doi: 10.1007/s10461-017-1726-5

Dawson, A. S. (2015). Salvador Roquet, Maria Sabina, and the Trouble with Jipis. Hahr-Hispanic American Historical Review, 95(1), 103-133. doi: $\underline{10.1215 / 00182168-2836928}$

De Boni, R. B., Shepherd, B. E., Grinsztejn, B., Cesar, C., Cortes, C., Padgett, D., ... McGowan, C. C. (2016). Substance Use and Adherence Among People Living with HIV/AIDS Receiving CART in Latin America. Aids and Behavior, 20(11), 2692-2699. doi: 10.1007/s10461-016-1398-6

Díaz-Negrete, D. B., Rodríguez-Kuri, S. E., Gutiérrez-López, A. D., SánchezHuesca, R., \& Fernández-Cáceres, C. (2017). Trends and patterns of marijuana use in men and women seeking drug treatment. Salud Mental, 40(3), 103-110. doi: 10.17711/sm.0185-3325.2017.013

Duran-Martinez, A. (2015). Drugs Around the Corner: Domestic Drug Markets and Violence in Colombia and Mexico. Latin American Politics and Society, 57(3), 122-146. doi: 10.1111/j.1548$\underline{2456.2015 .00274 . x}$

Enciso, F. (2015). Nuestra historia narcótica: pasajes para (re) legalizar las drogas en México. Penguin Random House.

Gaines, T. L., Rudolph, A. E., Brouwer, K. C., Strathdee, S. A., Lozada, R., Martinez, G., ... Rusch, M. L. A. (2013). The longitudinal association of venue stability with consistent condom use among female sex workers in two Mexico-USA border cities. International Journal of STD \& AIDS, 24(7), 523-529. doi: 10.1177/0956462412473890

Gaines, T.L., Beletsky, L., Arredondo, J., Werb, D., Rangel, G., Vera, A., \& Brouwer, K. (2015). Examining the Spatial Distribution of Law Enforcement Encounters among People Who Inject Drugs after Implementation of Mexico's Drug Policy Reform. Journal of Urban Health, 92(2), 338-351. doi: 10.1007/s11524-014-9907-2

Gaines, Tommi L., Urada, L. A., Martinez, G., Goldenberg, S. M., Rangel, G., Reed, E., ... Strathdee, S. A. (2015). Short-term cessation of sex work and injection drug use: Evidence from a recurrent event survival analysis. Addictive Behaviors, 45, 63-69. doi: 10.1016/j.addbeh.2015.01.020

Gaines, Tommi L., Werb, D., Arredondo, J., Alaniz, V. M., Vilalta, C., \& Beletsky, L. (2017). The Spatial-Temporal Pattern of Policing Following a Drug Policy Reform: Triangulating Self-Reported Arrests
With Official Crime Statistics. Substance Use \& Misuse, 52(2), 214-222. doi: $10.1080 / 10826084.2016 .1223689$

Garcia, A. (2015). Serenity: Violence, Inequality, and Recovery on the Edge of Mexico City. Medical Anthropology Quarterly, 29(4), 455-472. doi: $\underline{10.1111 / \mathrm{maq} .12208}$

Garcia, Angela, \& Anderson, B. (2016). Violence, addiction, recovery: An anthropological study of Mexico's anexos. Transcultural Psychiatry, 53(4), 445-464. doi: 10.1177/1363461516662539

García Aurrecoechea, V. R., Rodríguez Kuri, S. E., Córdova Alcaráz, A. J., \& Fernández Cáceres, M. del C. (2016). Diagnóstico macrosocial de riesgo del consumo de drogas en México. Acta de Investigación Psicológica, 6(3), 2516-2526. doi: 10.1016/j.aipprr.2016.11.005

Gelberg, L., Natera Rey, G., Andersen, R. M., Arroyo, M., BojorquezChapela, I., Rico, M. W., ... Serota, M. (2017). Prevalence of Substance Use Among Patients of Community Health Centers in East Los Angeles and Tijuana. Substance Use \& Misuse, 52(3), 359-372. doi: $\underline{10.1080 / 10826084.2016 .1227848}$

Genet Guzmán Chávez, M. (2013). El culto del Santo Daime. Apuntes para la legalización del uso de sustancias psicoactivas en contextos ceremoniales en México. Revista de El Colegio de San Luis, II/(5), 5789.

Giacomello, C. (2017). Women and drug policies in Latin america: A critical review of the united nations resolution 'mainstreaming a gender perspective in drug-related policies and programmes'. Howard Journal of Crime and Justice, 56(3), 288-308. doi: 10.1111/hojo.12216

Gigengack, R. (2014). The chemo and the mona: Inhalants, devotion and street youth in Mexico City. International Journal of Drug Policy, 25(1), 61-70. doi: 10.1016/j.drugpo.2013.08.001

Gigengack, R. (2016). Chapter 99 - Inhalant Drug Use and Street Youth: Ethnographic Insights from Mexico City**Parts of this chapter are based upon Gigengack (2014a). With permission from Elsevier. En V. R. Preedy (Ed.), Neuropathology of Drug Addictions and Substance Misuse (pp. 1075-1084). San Diego: Academic Press. doi: 10.1016/B978-0-12-800212-4.00099-6

Gómez San Luis, A. H., \& Almanza Avendaño, A. M. (2016). Impacto del narcotráfico en jóvenes de Tamaulipas, México: Drogas e inseguridad. [Impact of drug trafficking in young adults from Tamaulipas, Mexico: Drugs and insecurity.]. Revista de Psicología, 34(2), 445-472. doi: 10.18800/psico.201602.009

González-Forteza, C., Juárez López, C. E., Montejo León, L. de los Á., Oseguera Díaz, G., Wagner Echeagaray, F. A., \& Jiménez Tapia, A. (2015). Ideación suicida y su asociación con drogas, depresión e impulsividad en una muestra representativa de estudiantes de secundaria del estado de Campeche, México. Acta Universitaria, 25(2), 29-34.

Gracia-Gutiérrez de Velasco, S. E., Díaz-Negrete, D. B., \& FernándezCáceres, C. (2015). Elaboración metafórica en una intervención narrativoconversacional de tratamiento del uso de drogas. Revista de Psicología, 24(2), 1-18.

Guerrero, E. G., Villatoro, J. A., Kong, Y., Fleiz, C., Vega, W. A., Strathdee, S. A., \& Medina-Mora, M. E. (2014). Barriers to accessing substance 
abuse treatment in Mexico: National comparative analysis by migration status. Substance Abuse Treatment, Prevention, and Policy, 9.

Guerrero, E. G., Villatoro, J. A., Kong, Y., Gamiño, M. B., Vega, W. A., \& Medina-Mora, M. E. (2014). Mexicans' use of illicit drugs in an era of drug reform: National comparative analysis by migrant status. International Journal of Drug Policy, 25(3), 451-457. doi: 10.1016/j.drugpo.2014.04.006

Guimaraes Borges, G. L., Mendoza Meléndez, M. Á., López Brambila, M. Á., García Pacheco, J. Á., Velasco-Ángeles, L. R., Beltrán Silva, M. A., ... Camacho Solís, R. (2014). Prevalencia y factores asociados al consumo de tabaco, alcohol y drogas en una muestra poblacional de adultos mayores del Distrito Federal. Salud mental, 37(1), 15-25.

Guzmán Chávez, M. G. (2016). Why peyote must be valued as biocultural patrimony of Mexico. En Peyote: History, tradition, politics, and conservation. (pp. 239-263). Santa Barbara, CA, US: Praeger/ABCCLIO.

Guzmán Facundo, F. R., García Salas, B. A., Rodríguez Aguilar, L., \& Alonso Castillo, M. M. (2014). Actitud, norma subjetiva y control conductual como predictores del consumo de drogas en jóvenes de zona marginal del norte de México. Frontera Norte, 26(51), 53-74.

Harvey-Vera, A. Y., González-Zúñiga, P., Vargas-Ojeda, A. C., Medina-Mora, M. E., Magis-Rodríguez, C. L., Wagner, K., ... Werb, D. (2016). Risk of violence in drug rehabilitation centers: Perceptions of people who inject drugs in Tijuana, Mexico. Substance Abuse Treatment, Prevention, and Policy, 11. doi: 10.1186/s13011-015-0044-z

Hernández González, S., \& Sotelo Morales, J. (2013). Argumentos para el debate sobre la legalización de la marihuana en México. Entreciencias: Diálogos en la Sociedad del Conocimiento, 1(2), 93-100.

Hiller, S. P., Syvertsen, J. L., Lozada, R., \& Ojeda, V. D. (2013). Social support and recovery among Mexican female sex workers who inject drugs. Journal of Substance Abuse Treatment, 45(1), 44-54. doi: 10.1016/j.jsat.2012.12.009

Horigian, V. E., Espinal, P. S., Alonso, E., Verdeja, R. E., Duan, R., Usaga, I. M., ... Feaster, D. J. (2016). Readiness and barriers to adopt evidencebased practices for substance abuse treatment in Mexico. Salud Mental, 39(2), 77-84. doi: 10.17711/SM.0185-3325.2016.004

Horyniak, D., Pinedo, M., Burgos, J. L., \& Ojeda, V. D. (2017). Relationships between integration and drug use among deported migrants in Tijuana, Mexico. Journal of Immigrant and Minority Health, 19(5), 1196-1206. doi: 10.1007/s10903-016-0518-8

Horyniak, D., Strathdee, S. A., West, B. S., Meacham, M., Rangel, G., \& Gaines, T. L. (2018). Predictors of injecting cessation among a cohort of people who inject drugs in Tijuana, Mexico. Drug and Alcohol Dependence, 185, 298-304. doi: 10.1016/j.drugalcdep.2017.12.034

Kalkach-Aparicio, M., Cuéllar-Herrera, M., Flores-Ramírez, E. L., RuízGadea, P., Medina-Osti, L., Trejo-Martínez, D., ... GorianMontealegre, G. (2016). The use of cannabis as an antiepileptic treatment in Mexico: A review, bioethical analysis, discussion and position of the Hospital General de México Epilepsy Clinic. Revista Médica del Hospital General de México, 79(2), 68-78. doi: 10.1016/j.hgmx.2016.04.004
Kori, N., Roth, A. M., Lozada, R., Vera, A., \& Brouwer, K. C. (2014). Correlates of injecting in an HIV incidence hotspot among substance users in Tijuana, Mexico. International Journal of Drug Policy, 25(3), 525-532. doi: 10.1016/j.drugpo.2013.12.005

Kulis, S., Booth, J. M., \& Becerra, D. (2016). Drug-Resistance Strategies of Early Adolescents in Mexico: Gender Differences in the Influence of Drug Offers and Relationship to the Offeror. Substance Use and Misuse, 51(3), 370-382. doi: 10.3109/10826084.2015.1110171

Labate, B. C., \& Feeney, K. (2016). Paradoxes of peyote regulation in Mexico: Drug conventions and environmental laws. En Peyote: History, tradition, politics, and conservation. (pp. 211-238). Santa Barbara, CA, US: Praeger/ABC-CLIO.

Labate, B. C., \& Ruiz Flores López, P. (2014). Critical reflections on the National Addiction Surveys (ENAs) in Mexico. Drugs: Education, Prevention \& Policy, 21(6), 427-433. doi: $\underline{10.3109 / 09687637.2014 .938022}$

Labate, B., Cavnar, C., \& Rodrigues, T. (2016). Drug policies and the politics of drugs in the Americas (Springer). Switzerland: Springer International Publishing. doi: 10.1007/978-3-319-29082-9_2

Leon, N. (2016). The prevalence and determinants of health risk behaviors among high school students in Mexico. Loma Linda University, US.

Loza, O., Ramos, R., Ferreira-Pinto, J., Hernandez, M. T., \& Villalobos, S. A. (2016). A qualitative exploration of perceived gender differences in methamphetamine use among women who use methamphetamine on the Mexico-U.S. border. Journal of Ethnicity in Substance Abuse, 15(4), 405-424. doi: 10.1080/15332640.2015.1070392

Lozano-Verduzco, I., Marín-Navarrete, R., Romero-Mendoza, M., \& TenaSuck, A. (2015). Experiences of Power and Violence in Mexican Men Attending Mutual-Aid Residential Centers for Addiction Treatment. American Journal of Men's Health, 10(3), 237-249. doi: $\underline{10.1177 / 1557988314565812}$

Lozano-Verduzco, Ignacio, Romero-Mendoza, M., \& Marín-Navarrete, R. (2016). Violence narratives of Mexican women treated in mutual-aid residential centers for addiction treatment. Substance Abuse Treatment, Prevention, and Policy, 11.

Luna-Fabritius, A. (2015). Modernidad y drogas desde una perspectiva histórica. Revista Mexicana de Ciencias Políticas y Sociales, LX(225), $27-$ 50.

Mañón Garibay, G. J. (2017). The Consumption of Marijuana from A Legal and Philosophical Viewpoint. Mexican Law Review, 10(1), 129-142. doi: 10.22201/iij.24485306e.2017.19.11386

Marín-Navarrete, R., Fernández-Mondragón, J., Eliosa-Hernández, A., Nuñez, L. T., Graue-Moreno, J., Galván-Sosa, D., ... Berlanga, C. (2013). Consideraciones metodológicas y éticas para la conducción de ensayos clínicos controlados aleatorizados (ECCA) sobre intervenciones para las adicciones. Salud Mental, 36(3), 253-265. doi: 10.17711/SM.0185-3325.2013.032

Marin-Navarrete, R., Horigian, V. E., Medina-Mora, M. E., Verdeja, R. E., Alonso, E., Feaster, D. J., ... De la Fuente-Martin, A. (2017). Motivational enhancement treatment in outpatient addiction 
centers: A multisite randomized trial. International Journal of Clinical and Health Psychology, 17(1), 9-19. doi: 10.1016/j.ijchp.2016.05.001

Marín-Navarrete, R., Templos-Nuñez, L., Eliosa-Hernández, A., VillalobosGallegos, L., Fernández-Mondragón, J., Pérez-López, A., ... Horigian, V. E. (2014). Characteristics of a treatment-seeking population in outpatient addiction treatment centers in Mexico. Substance Use and Misuse, 49(13), 1784-1794. doi: 10.3109/10826084.2014.931972

Marsiglia, F. F., Booth, J. M., Ayers, S. L., Nuño-Gutierrez, B. L., Kulis, S., \& Hoffman, S. (2014). Short-term effects on substance use of the Keepin' It REAL Pilot Prevention Program: Linguistically adapted for youth in Jalisco, Mexico. Prevention Science, 15(5), 694-704. doi: $\underline{10.1007 / s 11121-013-0421-7}$

Marsiglia, F. F., Kulis, S. S., Booth, J. M., Nuño-Gutierrez, B. L., \& Robbins, D. E. (2015). Long-term effects of the keepin' it REAL model program in Mexico: Substance use trajectories of Guadalajara middle school students. The Journal of Primary Prevention, 36(2), 93-104. doi: 10.1007/s10935-014-0380-1

Martínez Martínez, K. I., Pacheco Trejo, A. Y., Echeverría San Vicente, L., \& Medina-Mora, M. E. (2016). Barriers to technology transfer: A qualitative study of brief interventions and the addiction treatment centers. Salud Mental, 39(5), 257-265. doi: 10.17711/SM.0185$\underline{3325.2016 .030}$

Martinez Valenzuela, C. (2013). The "War on Drugs" and the "New Strategy": Identity constructions of the United States, U.S. drug users and Mexico. Mexican Law Review, 5(2), 245-275. doi: 10.1016/S1870-0578(16)30025-7

Martínez Vélez, N. A., Sánchez Hernández, G. Y., Vázquez Pérez, L., \& Tiburcio Sainz, M. A. (2016). Las aportaciones de 40 años de investigación epidemiológica en México sobre consumo de solventes inhalables. [Contributions from 40 years of epidemiological research on inhalant use in Mexico.]. Salud Mental, 39(2), 85-97. doi: 10.17711/SM.0185-3325.2016.005

Matsui-Santana, O. J., Alcalá-Fonseca, J. C., Calvo-Cruz, R. J. L., RojasAranda, A. G., Villaseñor-Farías, M., Zárate-Lazcano, C., ... Tello-Vaca, C. E. (2017). Child Sexual Abuse in Male Drug Addicts of Guadalajara, Mexico. 23rd Congress of the World Association for Sexual Health, 14(5, Supplement 4), e344. doi: 10.1016/j.jsxm.2017.04.624

McDougal, L., Strathdee, S. A., Rangel, G., Martinez, G., Vera, A., Sirotin, N., ... Raj, A. (2013). Adverse pregnancy outcomes and sexual violence among female sex workers who inject drugs on the United StatesMexico border. Violence and Victims, 28(3), 496-512. doi: $\underline{10.1891 / 0886-6708.11-00129}$

Meacham, M.C., Roesch, S. C., Strathdee, S. A., Lindsay, S., GonzalezZuniga, P., \& Gaines, T. L. (2018). Latent classes of polydrug and polyroute use and associations with human immunodeficiency virus risk behaviours and overdose among people who inject drugs in Tijuana, Baja California, Mexico. Drug and Alcohol Review, 37(1), 128-136. doi: 10.1111/dar.12524

Meacham, M.C., Rudolph, A. E., Strathdee, S. A., Rusch, M. L., Brouwer, K. C., Patterson, T. L., ... Roesch, S. C. (2015). Polydrug Use and HIV Risk among People Who Inject Heroin in Tijuana, Mexico: A Latent Class Analysis. Substance Use and Misuse, 50(10), 1351-1359. doi: $\underline{10.3109 / 10826084.2015 .1013132}$
Meacham, Meredith C., Roesch, S. C., Strathdee, S. A., \& Gaines, T. L. (2018). Perceived Treatment Need and Latent Transitions in Heroin and Methamphetamine Polydrug Use among People who Inject Drugs in Tijuana, Mexico. Journal of Psychoactive Drugs, 50(1), 62-71. doi: 10.1080/02791072.2017.1370747

Meacham, Meredith C., Strathdee, S. A., Rangel, G., Armenta, R. F., Gaines, T. L., \& Garfein, R. S. (2016). Prevalence and Correlates of HeroinMethamphetamine Co-Injection Among Persons Who Inject Drugs in San Diego, California, and Tijuana, Baja California, Mexico. Journal of Studies on Alcohol and Drugs, 77(5), 774-781. doi: 10.15288/jsad.2016.77.774

Mejía Cruz, D., Morales Chainé, S., \& Nieto Gutiérrez, J. (2015). Descuento Temporal Asociado Al Uso De Cocaína. Revista Mexicana de Análisis de la Conducta, 41(1), 86-101.

Melo, J. S., Garfein, R. S., Hayashi, K., Milloy, M. J., DeBeck, K., Sun, S., ... Werb, D. (2018). Do law enforcement interactions reduce the initiation of injection drug use? An investigation in three North American settings. Drug and Alcohol Dependence, 182, 67-73. doi: 10.1016/j.drugalcdep.2017.10.009

Mendiburo-Seguel, A., Vargas, S., Oyanedel, J. C., Torres, F., Vergara, E., \& Hough, M. (2017). Attitudes towards drug policies in Latin America: Results from a Latin-American Survey. International Journal of Drug Policy, 41, 8-13. doi: 10.1016/j.drugpo.2016.10.001

Mendoza-Meléndez, M. A., Guimaraes, G. L. B., Gallegos-Cari, A., Pacheco, J. Á. G., Hernández-Llanes, N. F., Camacho-Solís, R., \& Medina-Mora Icaza, M. E. (2015). Asociación del consumo de sustancias psicoactivas con el cuidado y la salud del adulto mayor. [Association of psychoactive substances use with care and health of older adults.]. Salud Mental, 38(1), 15-26. doi: 10.17711/SM.0185$\underline{3325.2015 .002}$

Mercado Maldonado, A., \& Briseño Cruz, P. D. (2014). El "yo" deteriorado: estigma y adicción en la sociedad del consumo. Espacios Públicos, 17(39), 137-157.

Mora-Rios, J., Ortega-Ortega, M., \& Elena Medina-Mora, M. (2017). Addiction-Related Stigma and Discrimination: A Qualitative Study in Treatment Centers in Mexico City. Substance Use \& Misuse, 52(5), 594-603. doi: 10.1080/10826084.2016.1245744

Morris, M. D., Lemus, H., Wagner, K. D., Martinez, G., Lozada, R., Gómez, R. M., \& Strathdee, S. A. (2013). Factors associated with pathways toward concurrent sex work and injection drug use among female sex workers who inject drugs in Northern Mexico. Addiction, 108(1), 161-170. doi: 10.1111/j.1360-0443.2012.04016.x

Negi, N., Valdez, A., Cepeda, A., \& Natera, G. (2015). Patterns of crack use among drug-using females in Mexico city. Drug and Alcohol Dependence, 146, e49. doi: 10.1016/j.drugalcdep.2014.09.505

Nowotny, K. M., Cepeda, A., Perdue, T., Negi, N., \& Valdez, A. (2017). Risk environments and substance use among Mexican female sex work on the U.S.-Mexico border. Journal of Drug Issues, 47(4), 528-542. doi: $10.1177 / 0022042616678609$

Núñez Valadez, M. A. (2014). Drug Use and the Right to Health: An Analysis of International Law and the Mexican Case. Mexican Law Review, 6(2), 201-224. 
Orantes López, C., Ayala Guerrero, F., Méndez Ubach, M., Oropeza Tena, R., \& Juárez García, F. L. (2013). Arquitectura de sueño en dependientes de la cocaína en abstinencia crónica. Psicología Iberoamericana, 21(2), 48-59.

Oropeza Tena, R., Lira Mandujano, J., \& Rosales García, M. (2013). Perfil sociodemográfico y patrones de consumo de drogas de pacientes de cocaina y crack. Diversitas: Perspectivas en Psicología, 9(2), 373382.

Ortiz Castro, A., Domínguez García, M. J., \& Palomares Calderón, G. (2015). El consumo de solventes inhalables en la festividad de San Judas Tadeo. [Solvent inhalants use in the San Judos Tadeo feast.]. Salud Mental, 38(6), 427-432. doi: 10.17711/SM.0185-3325.2015.057

Ortiz Castro, A., Meza, D., \& Martínez, R. (2014). Poppers, una droga emergente. Resultados del Sistema de Reporte de Informacion en Drogas. [Poppers, an emerging drug. Results reporting system in drug information.]. Salud Mental, 37(3), 225-231. doi: 10.17711/SM.0185-3325.2014.026

Paley, D. (2017). STATE POWER AND THE ENFORCEMENT OF PROHIBITION IN MEXICO. Mexican Law Review, 10(1), 3-20. doi: $\underline{10.22201 / \text { iij.24485306e.2017.19.11381 }}$

Palinkas, L. A., Bazzi, A. R., Syvertsen, J. L., Ulibarri, M. D., Hernandez, D., Rangel, M. G., ... Strathdee, S. A. (2016). Measuring current drug use in female sex workers and their noncommercial male partners in Mexico: Concordance between data collected from surveys versus semi-structured interviews. Substance Use and Misuse, 51(1), 23-33. doi: 10.3109/10826084.2015.1073326

Palinkas, L. A., Robertson, A. M., Syvertsen, J. L., Hernandez, D. O., Ulibarri, M. D., Rangel, M. G., ... Strathdee, S. A. (2014). Client perspectives on design and implementation of a couples-based intervention to reduce sexual and drug risk behaviors among female sex workers and their noncommercial partners in Tijuana and Ciudad Juárez, México. AIDS and Behavior, 18(3), 583-594. doi: 10.1007/s10461-014$\underline{0715-1}$

Patteson, J. W. (2018). A dialectics of intoxication: Addiction, violence, and vision in Latin American cultural production. The University of Wisconsin-Madison, US.

Pérez-Romero, L. Á., Quiroga-Anaya, H., \& Pérez-Romero, A. P. (2014). Supresión de pensamientos y días sin consumo de droga comovariables predictivas del craving. Revista Latinoamericana de Medicina Conductual / Latin American Journal of Behavioral Medicine, $4(2), 83-92$.

Pinedo Banuelos, M. (2016). Social and structural conditions of deportation that influence HIV risk among Mexican deportees who inject drugs in the U.S-Mexico border. UC San Diego, US.

Pinedo, M., Beletsky, L., Alamillo, N., \& Ojeda, V. D. (2017). Healthdamaging policing practices among persons who inject drugs in Mexico: Are deported migrants at greater risk? International Journal of Drug Policy, 46, 41-46. doi: 10.1016/j.drugpo.2017.05.028

Pinedo, M., Burgos, J. L., Robertson, A. M., Vera, A., Lozada, R., \& Ojeda, V. D. (2014). Perceived risk of HIV infection among deported male injection drug users in Tijuana, Mexico. Global Public Health: An
International Journal for Research, Policy and Practice, 9(4), 436-454. doi: $10.1080 / 17441692.2014 .893367$

Pinedo, M., Burgos, J. L., Zuniga, M. L., Perez, R., Macera, C. A., \& Ojeda, V. D. (2015). Police Victimization Among Persons Who Inject Drugs Along the US-Mexico Border. Journal of Studies on Alcohol and Drugs, 76(5), 758-763.

Pinedo, M., Kang Sim, D. E., Espinoza Giacinto, R., \& Zuñiga, M. L. (2016). An exploratory study of internal migration and substance use among an indigenous community in Southern Mexico. Family \& Community Health: The Journal of Health Promotion \& Maintenance, 39(1), 24-30. doi: 10.1097/FCH.0000000000000085

Pitpitan, E. V., Patterson, T. L., Abramovitz, D., Vera, A., Martinez, G., Staines, H., \& Strathdee, S. A. (2016). Policing Behaviors, Safe Injection Self-Efficacy, and Intervening on Injection Risks: Moderated Mediation Results From a Randomized Trial. Health Psychology, 35(1), 87-91. doi: 10.1037/hea0000241

Pollini, R. A., Gallardo, M., Ruiz, S., Case, P., Zaller, N., \& Lozada, R. (2014). Over-the-counter but out of reach: a pharmacy-based survey of OTC syringe sales in Tijuana, Mexico. Journal of Health Care for the Poor and Underserved, 25(2), 637-651. doi: 10.1353/hpu.2014.0090

Rafful, C., Wagner, K. D., Werb, D., González-Zúñiga, P. E., Verdugo, S., Rangel, G., \& Strathdee, S. A. (2015). Prevalence and correlates of neck injection among people who inject drugs in Tijuana, Mexico. Drug and Alcohol Review, 34(6), 630-636. doi: 10.1111/dar.12264

Rafful, Claudia, Melo, J., Medina-Mora, M. E., Rangel, G., Sun, X., Jain, S., \& Werb, D. (2017). Cross-border migration and initiation of others into drug injecting in tijuana, mexico. Drug and Alcohol Review, No Pagination Specified-No Pagination Specified. doi: $\underline{10.1111 / \text { dar. } 12630}$

Rafful, Claudia, Mittal, M. L., Melo, J. S., Werb, D., \& Guise, A. (2017). Initiation into Injection Drug Use and Cross-Border Migration Among People Who Inject Drugs in Tijuana, Mexico. Annals of Behavioral Medicine, 51, S1397-S1398.

Rafful, Claudia, Orozco, R., Rangel, G., Davidson, P., Werb, D., Beletsky, L., \& Strathdee, S. A. (2018). Increased non-fatal overdose risk associated with involuntary drug treatment in a longitudinal study with people who inject drugs. Addiction, No Pagination Specified-No Pagination Specified. doi: 10.1111/add.14159

Rafful, Claudia, Zule, W., Gonzalez-Zuniga, P. E., Werb, D., Medina-Mora, M. E., Magis-Rodriguez, C., \& Strathdee, S. A. (2015). High deadspace syringe use among people who inject drugs in Tijuana, Mexico. American Journal of Drug and Alcohol Abuse, 41(3), 220-225. doi: 10.3109/00952990.2015.1011742

Rane, A., Church, S., Bhatia, U., Orford, J., Velleman, R., \& Nadkarni, A. (2017). Psychosocial interventions for addiction-affected families in Low and Middle Income Countries: A systematic review. Addictive Behaviors, 74, 1-8. doi: 10.1016/j.addbeh.2017.05.015

Reveles, J. (2013). SOCIAL AND ECONOMIC DAMAGE CAUSED BY THE WAR AGAINST DRUGS IN MEXICO. (R. Schonenberg \& HeinrichBollStiftung, Eds.) (Vol. 17). 
Ribeiro Schneider, D., Vidal-Infer, A., Bolaños-Pizarro, M., AleixandreBenavent, R., Bueno Cañigral, F. J., \& Valderrama-Zurián, J. C. (2014). Colaboración científica en drogodependencias entre Latino América y la Unión Europea (2001-2010) a partir de la ISI Web of Science. [Scientific cooperation on drug abuse between Latin American and the European Union (2001-2010) from the ISI Web of Science.]. Salud Mental, 37(3), 205-216. doi: 10.17711/SM.01853325.2014.024

Robertson, A. M., Garfein, R. S., Wagner, K. D., Mehta, S. R., MagisRodriguez, C., Cuevas-Mota, J., ... Strathdee, S. A. (2014). Evaluating the impact of Mexico's drug policy reforms on people who inject drugs in Tijuana, B. C., Mexico, and San Diego, CA, United States: A binational mixed methods research agenda. Harm Reduction Journal, 11.

Robles-García, R., Fresán, A., Castellanos-Ryan, N., Conrod, P., Gómez, D., de Quevedo y Domínguez, M. E. G., ... Medina-Mora, M. E. (2014). Spanish version of the Substance Use Risk Profile Scale: Factor structure, reliability, and validity in Mexican adolescents. Psychiatry Research, 220(3), 1113-1117. doi: 10.1016/j.psychres.2014.08.057

Rodriguez-Montejano, S., Ojeda, V. D., Valles-Medina, A. M., \& VargasOjeda, A. (2015). Acculturative trajectories descriptions and implications for health among 12 Mexican deported women who inject drugs. Salud Mental, 38(6), 409-416.

Romo-González, T., \& Larralde, C. (2013). ¿Cuáles y qué tan graves son los daños a la salud individual y a la sociedad causados por el consumo de drogas lícitas e ilícitas? Psychologia. Avances de la disciplina, 7(1), 67-80.

Ruiz Martínez, A. O., Hernández Cera, M. I., Mayrén Arévalo, P. J. de J., \& Vargas Santillán, M. D. L. (2014). Funcionamiento Familiar De Consumidores De Sustancias Adictivas Con Y Sin Conducta Delictiva. Liberabit. Revista de Psicología, 20(1), 109-119.

Salas-García, B., De-San-Jorge-Cárdenas, X., Beverido-Sustaeta, P., \& Carmona-Avendaño, Y. (2016). La migración interna en estudiantes universitarios. ¿Un riesgo para el consumo de drogas? CienciaUAT, 10(2), 23-32.

Sánchez-Sosa, J. C., Villarreal-González, M. E., Ávila Guerrero, M. E., Jiménez, A. V., \& Musitu, G. (2014). Contextos de socializacion y consumo de drogas ilegales en adolescentes escolarizados. [Socialization contexts and illegal drug use among schooled adolescents.]. Psychosocial Intervention, 23(1), 69-78. doi: $\underline{10.5093 / \mathrm{in} 2014 \mathrm{a} 7}$

Seidman, D., Rusch, M., Abramovitz, D., Stockman, J. K., Martinez, G., Rangel, G., ... Strathdee, S. A. (2016). Intravaginal practices among HIV-negative female sex workers along the US-Mexico border and their implications for emerging HIV prevention interventions. International Journal of Gynecology \& Obstetrics, 133(2), 212-216. doi: $\underline{10.1016 / j . i j g o .2015 .09 .025}$

Servin, A. E., Strathdee, S., Munoz, F. A., Vera, A., Rangel, G., \& Silverman, J. G. (2015). Vulnerabilities faced by the children of sex workers in two Mexico-US border cities: a retrospective study on sexual violence, substance use and HIV risk. Aids Care-Psychological and SocioMedical Aspects of Aids/Hiv, 27(1), 1-5. doi: $\underline{10.1080 / 09540121.2014 .946384}$
Silverman-Retana, O., Serván-Mori, E., McCoy, S. I., Larney, S., \& BautistaArredondo, S. (2017). Hepatitis c antibody prevalence among Mexico City prisoners injecting legal and illegal substances. Drug and Alcohol Dependence, 181, 140-145. doi: 10.1016/j.drugalcdep.2017.09.026

Smith, D. M., Werb, D., Abramovitz, D., Magis-Rodriguez, C., Vera, A., Patterson, T. L., \& Strathdee, S. A. (2016). Predictors of needle exchange program utilization during its implementation and expansion in Tijuana, Mexico. American Journal on Addictions, 25(2), 118-124. doi: 10.1111/ajad.12326

Syvertsen, Jennifer L., \& Bazzi, A. R. (2015). Sex Work, Heroin Injection, and HIV Risk in Tijuana: A Love Story. Anthropology of Consciousness, 26(2), 182-194. doi: 10.1111/anoc.12037

Syvertsen, Jennifer L., Bazzi, A. R., Martinez, G., Rangel, M. G., Ulibarri, M. D., Fergus, K. B., ... Strathdee, S. A. (2015). Love, trust, and HIV risk among female sex workers and their intimate male partners. American Journal of Public Health, 105(8), 1667-1674. doi: 10.2105/AJPH.2015.302620

Syvertsen, Jennifer L., Bazzi, A. R., \& Mittal, M. L. (2017). Hope Amidst Horror: Documenting the Effects of the «War On Drugs» Among Female Sex Workers and Their Intimate Partners in Tijuana, Mexico. Medical Anthropology, 36(6), 566-583. doi: $\underline{10.1080 / 01459740.2017 .1317770}$

Syvertsen, Jennifer L., Robertson, A. M., Palinkas, L. A., Gudelia Rangel, M., Martinez, G., \& Strathdee, S. A. (2013). «Where sex ends and emotions begin": love and HIV risk among female sex workers and their intimate, non-commercial partners along the Mexico-US border». Culture Health \& Sexuality, 15(5), 540-554. doi: 10.1080/13691058.2013.773381

Syvertsen, Jennifer L., Robertson, A. M., Strathdee, S. A., Martinez, G., Rangel, M. G., \& Wagner, K. D. (2014). Rethinking risk: Gender and injection drug-related HIV risk among female sex workers and their non-commercial partners along the Mexico-U.S. border. International Journal of Drug Policy, 25(5), 836-844. doi: 10.1016/j.drugpo.2014.02.005

Syvertsen, Jennifer Leigh. (2013). Love and risk: Intimate relationships among female sex workers who inject drugs and their non-commercial partners in Tijuana, Mexico. University of South Florida, US.

Thompson, G. O. (2014). Slowly Learning the Hard Way: U.S. America's War on Drugs And Implications for Mexico. Norteamérica, 9(2), 59-83. doi: 10.20999/nam.2014.b003

Tizoc-Marquez, A., Rivera-Fierro, K., Rieke-Campoy, U., \& Cruz-Palomares, M. (2017). Reducción de daños como estrategia para el uso y abuso de sustancias en México: desafios y oportunidades. Ra Ximhai, 13(2), 39-51.

Toquero Hernández, M. A., \& Salguero Velázquez, M. A. (2013). LOS SIGNIFICADOS DE SER HOMBRE ASOCIADOS AL CONSUMO DE SUSTANCIAS PSICOACTIVAS. Revista de Estudios de Género. La ventana, IV(38), 372-404.

Trujillo Bretón, J. A. (2017). The house of silence. Chinese and Mexicans involved in the illegal opium market and related activities in Guadalajara, Jalisco, from 1917 to 1950. Passagens-International 
Review of Political History and Legal Culture, 9(3), 416-445. doi: $\underline{10.15175 / 1984-2503-20179303}$

Ulibarri, M. D., Roesch, S., Rangel, M. G., Staines, H., Amaro, H., \& Strathdee, S. A. (2015). «Amar te Duele» («Love Hurts»): Sexual Relationship Power, Intimate Partner Violence, Depression Symptoms and HIV Risk Among Female Sex Workers Who Use Drugs and Their Non-commercial, Steady Partners in Mexico. Aids and Behavior, 19(1), 9-18. doi: 10.1007/s10461-014-0772-5

Valdez, A., Kaplan, C., Nowotny, K. M., Natera-Rey, G., \& Cepeda, A. (2015). Emerging patterns of crack use in Mexico City. International Journal of Drug Policy, 26(8), 739-745. doi: 10.1016/j.drugpo.2015.04.010

Valdez, A., Nowotny, K. M., Negi, N., Mora, E. Z., \& Cepeda, A. (2016). Un Jalon, Un Volteon, y Otra Vez: High-Risk Crack Smoking Paraphernalia in Mexico City. Journal of Psychoactive Drugs, 48(4), 295-302. doi: $10.1080 / 02791072.2016 .1198510$

Vilalta, C. J., \& Allmang, S. (2017). Assessing the Role of Context on the Relationship Between Adolescent Marijuana Use and Property Crimes in Mexico. Substance Use \& Misuse, 52(2), 152-163. doi: $\underline{10.1080 / 10826084.2016 .1222626}$

Villalobos-Gallegos, L., Pérez-López, A., Mendoza-Hassey, R., GraueMoreno, J., \& Marín-Navarrete, R. (2015). Psychometric and diagnostic properties of the Drug Abuse Screening Test (DAST): Comparing the DAST-20 vs. the DAST-10. Salud Mental, 38(2), 89-94.

Villatoro Velázquez, J.A., Bustos Gamiño, M. N., Fregoso Ito, D. A., Fleiz Bautista, C., Gutiérrez López, M. L., Amador Buenabad, N. G., \& Medina-Mora Icaza, M. E. (2017). Contextual factors associated with marijuana use in school population. Salud Mental, 40(3), 93-101. doi: 10.17711/SM.0185-3325.2017.012

Villatoro Velázquez, J.A., Medina-Mora Icaza, M. E., del Campo Sánchez, R. M., Fregoso Ito, D. A., Bustos Gamiño, M. N., Resendiz Escobar, E., ... Cañas Martínez, V. (2016). Drug use among students in Mexico: Trends and magnitude of the problem. Salud Mental, 39(4), 193-203. doi: 10.17711/SM.0185-3325.2016.023

Villatoro Velázquez, Jorge Ameth, Mendoza Meléndez, M. A., Moreno López, M. M., Oliva Robles, N., Fregoso Ito, D. F., Bustos Gamiño, M., ... Medina-Mora Icaza, M. E. (2014). Tendencias del uso de drogas en la Ciudad de México: Encuesta de estudiantes, octubre 2012. [Drug use trends in Mexico City: 2012 students survey.]. Salud Mental, 37(5), 423-435. doi: 10.17711/SM.0185-3325.2014.050

Volkmann, T., Fraga, M. A., Brodine, S. K., Iñiguez-Stevens, E., Cepeda, A., Elder, J. P., \& Garfein, R. S. (2013). Drug-scene familiarity and exposure to gang violence among residents in a rural farming community in Baja California, Mexico. Global Public Health: An International Journal for Research, Policy and Practice, 8(1), 65-78. doi: $\underline{10.1080 / 17441692.2012 .729220}$

Wagner, K. D., Pitpitan, E. V., Valente, T. W., Strathdee, S. A., Rusch, M., Magis-Rodriguez, C., ... Patterson, T. L. (2015). Place of Residence Moderates the Relationship Between Emotional Closeness and Syringe Sharing Among Injection Drug Using Clients of Sex Workers in the US-Mexico Border Region. Aids and Behavior, 19(6), 987-995. doi: 10.1007/s10461-015-1001-6
Wallisch, L., Zemore, S. E., Cherpitel, C. J., \& Borges, G. (2017). Wanting and getting help for substance problems on both sides of the US-Mexico border. Journal of Immigrant and Minority Health, 19(5), 1174-1185. doi: 10.1007/s10903-016-0442-y

Werb, D., Wagner, K. D., Beletsky, L., Gonzalez-Zuniga, P., Rangel, G., \& Strathdee, S. A. (2015). Police bribery and access to methadone maintenance therapy within the context of drug policy reform in Tijuana, Mexico. Drug and Alcohol Dependence, 148, 221-225. doi: 10.1016/j.drugalcdep.2015.01.011

Werb, Dan, Strathdee, S. A., Meza, E., Rangel Gomez, M. G., Palinkas, L., Medina-Mora, M. E., \& Beletsky, L. (2017). Institutional stakeholder perceptions of barriers to addiction treatment under Mexico's drug policy reform. Global Public Health: An International Journal for Research, Policy and Practice, 12(5), 519-530. doi: 10.1080/17441692.2015.1093524

Werb, Dan, Strathdee, S. A., Vera, A., Arredondo, J., Beletsky, L., GonzalezZuniga, P., \& Gaines, T. (2016). Spatial patterns of arrests, police assault and addiction treatment center locations in Tijuana, Mexico. Addiction, 111(7), 1246-1256. doi: 10.1111/add.13350

Werb, Daniel, Garfein, R., Kerr, T., Davidson, P., Roux, P., Jauffret-Roustide, M., ... Strathdee, S. A. (2016). A socio-structural approach to preventing injection drug use initiation: Rationale for the PRIMER study. Harm Reduction Journal, 13.

West, B. S., Abramovitz, D., Staines, H., Vera, A., Patterson, T. L., \& Strathdee, S. A. (2016). Predictors of Injection Cessation and Relapse among Female Sex Workers who Inject Drugs in Two Mexican-US Border Cities. Journal of Urban Health, 93(1), 141-154. doi: 10.1007/s11524-015-9995-7

Wood, E. F., Werb, D., Beletsky, L., Rangel, G., Cuevas Mota, J., Garfein, R. S., ... Wagner, K. D. (2017). Differential experiences of Mexican policing by people who inject drugs residing in Tijuana and San Diego. International Journal of Drug Policy, 41, 132-139. doi: 10.1016/j.drugpo.2016.12.010

Zhang, X., Martinez-Donate, A. P., Nobles, J., Hovell, M. F., Rangel, M. G., \& Rhoads, N. M. (2015). Substance use across different phases of the migration process: A survey of Mexican migrants flows. Journal of Immigrant and Minority Health, 17(6), 1746-1757. doi: 10.1007/s10903-014-0109-5 